\title{
Effects of methylprednisolone and MK-801 on functional recovery after experimental chronic spinal cord injury
}

\author{
SS Haghighi ${ }^{1}$, SK Agrawal ${ }^{*}, 1$, D Surdell, $\mathrm{Jr}^{1}$, R Plambeck ${ }^{1}, \mathrm{~S}$ Agrawal $^{2}, \mathrm{GC} \mathrm{Johnson}^{3}$ and A Walker ${ }^{3}$ \\ ${ }^{1}$ Section of Neurosurgery, University of Nebraska Medical Center, Omaha, Nebraska, USA; ${ }^{2}$ College of Nursing, \\ University of Nebraska Medical Center, Omaha, Nebraska, USA; ${ }^{3}$ Department of Veterinary Pathology, University \\ of Missouri-Columbia, Columbia, Missouri, USA
}

\begin{abstract}
Study design: An experimental study was conducted to evaluate the effects of methylprednisolone and MK-801 after the compressive injury of spinal cord in rats.

Objectives: To investigate the effect of methylprednisolone and non-competitive NMDA antagonist MK-801 in long-term functional outcome after spinal cord injury (SCI).

Methods: A randomized group A of Sprague-Dawley rats were treated with MK-801 $(1.0 \mathrm{mg} / \mathrm{kg}, n=10$; Group A) after a compression injury. A group of methylprednisolone (MP)-treated (30 mg/kg, $n=10$; Group B) and non-treated animals $(n=9$; Group C) were included for comparison. The functional motor outcome such as inclined plane (IP), toe spreading reflex (TSR), and modified Tarlov scale (TS) were measured in each animal at regular time points up to 8 weeks post-treatment. Histologically the injury site was scored in four groups and immunohistochemically Wallerian Degeneration (WD), astrocytosis and expression of $\beta$-amyloid protein was identified.

Results: In examining the IP data, no significant difference was recognized between the group means $(P$-value $>0.5)$. For the TSR, there were no differences in the group responses. For the $\mathrm{TS}$, the differences were not statistically significant. Only group B showed significance in cavitation scores compared to group A $(P>0.0094)$, WD was significantly different than group $\mathrm{C}(P>0.03)$, astrocytosis was significantly higher than group $\mathrm{A}(P>0.001)$ and modest presence of $\beta$-amyloid protein.

Conclusion: Our data indicate that one time bolus administration of MK-801 lacks any significant effect on axonal function in chronically injured rats. Daily bolus administration of $\mathrm{MP}$ at $30 \mathrm{mg} / \mathrm{kg}$ also did not ensure a better functional outcome. Immunohistochemically we have been able to show significant differences in WD, astrocytosis and small insignificant changes in $\beta$-amyloid protein.
\end{abstract}

Spinal Cord (2000) 38, $733-740$

Keywords: chronic spinal cord injury; methylprednisolone; MK 801; rats

\section{Introduction}

Within the last two decades, many investigations have focused on pathophysiology of acute spinal cord injury (SCI) in order to find methods to restore neurological function. ${ }^{1,2}$ It has been hypothesized that two interrelated mechanisms of damage to the spinal cord occur after injury: the primary mechanical injury and a subsequent secondary injury due to additional damaging processes, which follow the initial injury. ${ }^{3,4}$ The post-traumatic release of excitatory amino acids (EAA) and their actions on N-methyl-D-aspartate (NMDA) glutamate $(\mathrm{Glu})$ receptors appears to play an important role in the secondary injury process. ${ }^{5-7}$ NMDA significantly worsens trauma to the spinal cord. ${ }^{5}$ The

*Correspondence: SK Agrawal, Section of Neurosurgery, University of Nebraska Medical Center, 982035 Nebraska Medical Center, Omaha, Nebraska 68198-2035, USA ultimate damage to neurons occurs due to the rise in the intracellular ion concentrations in particular $\mathrm{Ca}^{2+}$ ions ${ }^{8,9}$ and high concentration of EAA such as Glu kill neurons. ${ }^{8}$ The neuronal damage caused by the early release of Glu and aspartate may be reduced by treatment with Glu release inhibitors or NMDA receptor antagonists. ${ }^{10,11}$

In the second and third National Acute Spinal Cord Injury Study (NASCIS) indicated that patients given methylprednisolone sodium succinate (MP) within $8 \mathrm{~h}$ of their injury had improved recovery of neurologic function. ${ }^{12,13}$ These studies provided clinical support for the concept of secondary injury process after traumatic injuries of spinal cord. In contrast, high dose MP failed to improve recovery of motor or sensory function at 6 weeks and 6 months if administration of MP was given $8 \mathrm{~h}$ or more after 
injury. ${ }^{12}$ However, recently Nesathurai raised concerns about the statistical analysis, randomization and clinical end points of NASCIS trials 2 and $3 .{ }^{14}$ This paper also raised the questionable clinical benefits and possible risks of MP. Similarly another recent review ${ }^{15}$ reported that high dose of MP in acute spinal cord injury does not improve neurological recovery.

In order to study the involvement of NMDA receptors and clinically proven methylprednisolone in SCI, we treated animals with the MP $(30 \mathrm{mg} / \mathrm{kg})$ and non-competitive NMDA antagonist MK801 ((+)-5methyl-10, 11- dihydro -5H -dibenzo[a,d]cyclohepten -5, 10 -imine) $(1 \mathrm{mg} / \mathrm{kg})$ after inducing a compressive SCI and compared with a non-treated group.

\section{Materials and methods}

\section{Surgical procedures and post operative care}

Thirty-two male rats (Harlan Sprague-Dawley Inc., Indianapolis, IN, USA) weighing $420 \pm 47 \mathrm{~g}$ were used. Animals were housed individually and maintained in a 12-h light/dark cycle. Isoflurane anesthesia was induced at $4 \%$ after placing the animals in a glass chamber. The anesthetized animals were transferred and fixed to a stereotactic frame (Model 1404, David Kopf Instruments, Tujunga, CA, USA., and were placed in a prone position on a heating blanket (Aquamatic Kmodel and K-pad, Gorman Rupp Industries, Belleville, $\mathrm{OH}$, USA). Body temperature was maintained between $36.5^{\circ} \mathrm{C}$ and $37.5^{\circ} \mathrm{C}$ (Model 401 , rectal thermometer, Yellow Springs Instruments Co, Yellow Springs, OH, USA). Isoflurane anesthesia was continued by mask. Heart rate was continuously monitored using an electrocardiograph machine (Model RS 3400, Gould Instruments, Cleveland, OH, USA).

Under sterile conditions, a midline incision at the lower thoracic level was performed. Under microscope, a T6-T9 dorsal laminectomy was performed. An aluminum compression device (weighing $5 \mathrm{~g}$ ) with a rectangular concave contact surface $(4 \times 4 \mathrm{~mm})$ and a round platform was positioned on the dorsal midline of the spinal cord using a micropositioner. The compressive injury was produced by placing a $45 \mathrm{~g}$ weight on the platform (total compression weight $=50 \mathrm{~g}$ ) for $2 \mathrm{~min}$. The compression produced a partial (incomplete) injury. After the injury, the wounds were closed in layers and the animals were placed into cages. Animals received ampicillin $50 \mathrm{mg} / \mathrm{Kg}$ IM before and every $6 \mathrm{~h}$ after the surgery up to $24 \mathrm{~h}$. The urinary bladder was expressed in each animal three times a day until full recovery of spontaneous micturition occurred.

\section{Drug administration}

Rats were randomly placed into three groups. Group A $(n=10)$ received a single injection of MK801 at $1 \mathrm{mg} /$ $\mathrm{kg}$ immediately after the injury. Group B $(n=10)$ received an intraperitoneal bolus dose of methylprednisolone $(30 \mathrm{mg} / \mathrm{kg})$ immediately after the injury and at
2, 3, 4, 5, 7 and 14 days post-injury. Control group C $(n=10)$ received an equal volume of bacteriostatic saline post-injury. Two sham operated rats underwent laminectomy and exposure of the thoracic spinal cord but they were not subjected to spinal cord injury and served as control for later histological evaluation.

\section{Behavioral assessment}

All animals were monitored for 8 weeks after the initial injury. During this period, each animal was subjected to a behavior examination using the inclined plane (IP), toe spreading reflex (TSR) and a modified Tarlov's scale (TS). The IP task measures the animal's ability to maintain its body position when placed on an inclined board. ${ }^{16}$ The TSR is elicited after picking up the animal and allowing the hindlimbs to hang free. ${ }^{17}$ The TS indicates the animal's hindlimb locomotor ability. ${ }^{18}$ Each animal was tested prior to the injury (Day 0) and at days 1, 3, 5, 7, 14, 21, 28, 35, 42, 49 and 56 post-injury. These tests were performed, in a blinded fashion, by a second investigator unaware of the treatment schedule.

\section{Histological preparation of spinal cord}

After termination of the treatment period (56th day post-injury), three animals in each group were selected for histology. These animals were sacrificed by intracardiac perfusion with $10 \%$ buffered formalin under deep level of pentobarbital anesthesia. Segments of spinal cord encased in their vertebrae were removed and fixed by immersion in neutral buffered formalin. Spinal segments were then decalcified for $24 \mathrm{~h}$ in HCI-EDTA solution (Surgipath Decalcifier II, Surgipath Medical Industries, Inc., Richmond, IL, USA). The segments were trimmed and cut in a dorsal plane at the level of the dorsal root ganglion and embedded in paraffin. Four series of $5 \mu \mathrm{m}$-thick sections were prepared at $100 \mu \mathrm{m}$ intervals, proceeding rostral to caudal in the cord. An HE-stained section was prepared from the most dorsal specimen at each of the four levels by standard methods and scored. ${ }^{19}$ Sections were evaluated using four criteria on light microscopic examination. Three of the criteria were designed to assess the amount of tissue destruction. Malacia, if present, was scored as being $0-4$ in severity, according to the estimated proportion of the specimen's width affected in the most severely affected section. The amount of Wallerian degeneration was evaluated by assessing the degree of vacuolation and axonal degeneration distal to the central area of malacia. The amount of axonal loss proximal to the central focus of malacia was evaluated by examining Sevier-Munger stained tissues. The sum of these three scores, averaged over the four step-sections examined in each animal, was used to summarize the overall degree of histological tissue damage in spinal cord sections. Scores for the individual sections were averaged and 
statistically analyzed by the Mann-Whitney Rank Sum Test. (Sigma Stat 1.0, SPSS, Chicago, IL, USA). Differences at the 0.05 level were considered significant.

After initial evaluation, randomized serial sections from the most severely affected level of each spinal cord were then stained immunohistochemically, in an effort to evaluate Wallerian degeneration (WD) by loss of myelin and axonal staining, astrocytosis, and expression of $\beta$-amyloid stress protein. Reagents used included single monoclonal antibodies or cocktail reagents directed at myelin basic protein (MBP, SMI 99, diluted 1:3000), glial fibrillary acidic protein (GFAP, SMI 22, diluted 1:500), and phosphorylated neurofilament epitopes (PNF, SMI 310, diluted $1: 500$; Sternberger Monoclonals Incorporated, Baltimore, MD, USA). $\beta$-amyloid detection utilized monoclonal antibody (clone $6 \mathrm{~F} / 3 \mathrm{D}$, diluted $1: 1000$, Dako, Carpenteria, CA, USA), which was applied to sections following a $5 \mathrm{~min}$ room temperature immersion in concentrated formic acid. Sections to be stained with GFAP-reagent were treated with Target
Unmasking Fluid (Signet Laboratories, Dedham, MA, USA) that had been heated to $90^{\circ} \mathrm{C}$, by immersing them for $5 \mathrm{~min}$. For staining, specimens were deparaffinized and peroxidase activity, pretreated as described above, washed, and blocked for nonspecific binding by treatment with goat serum. Sections were then incubated with the dilute monoclonal antibodies overnight at $4{ }^{\circ} \mathrm{C}$, washed, and sites of binding were identified using commercially available avidin-biotin-peroxidase kits (Vectastain ABC kit, Vector Labs, Burlingame, CA, USA). Vector Red was used as chromogen. Specimens were finally washed, counterstained with hematoxylin, dehydrated, cleared and mounted for microscopic examination.

\section{Statistical analysis}

In this study we were interested in the final functional outcome of the response variables. Repeated measure analysis of variance was done with one between subject (Group) and one with in subject (days) variable for
A

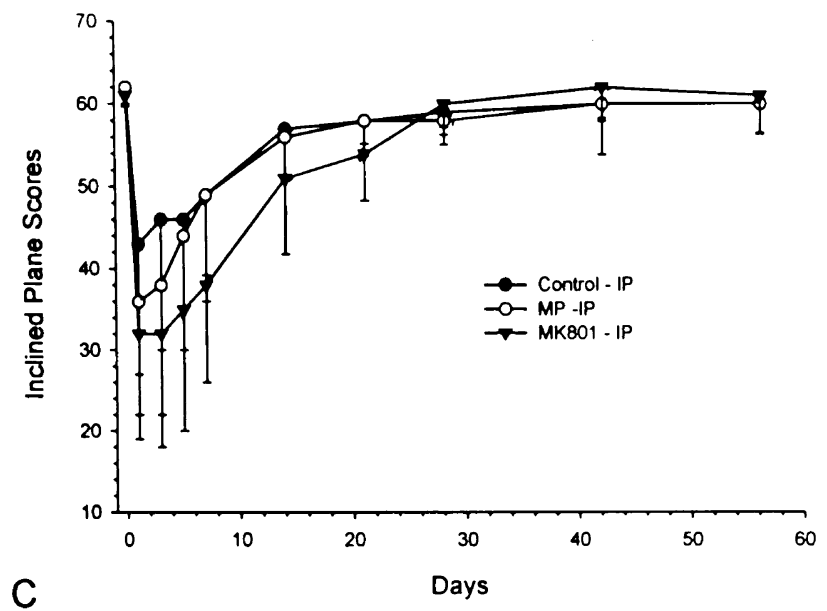

C

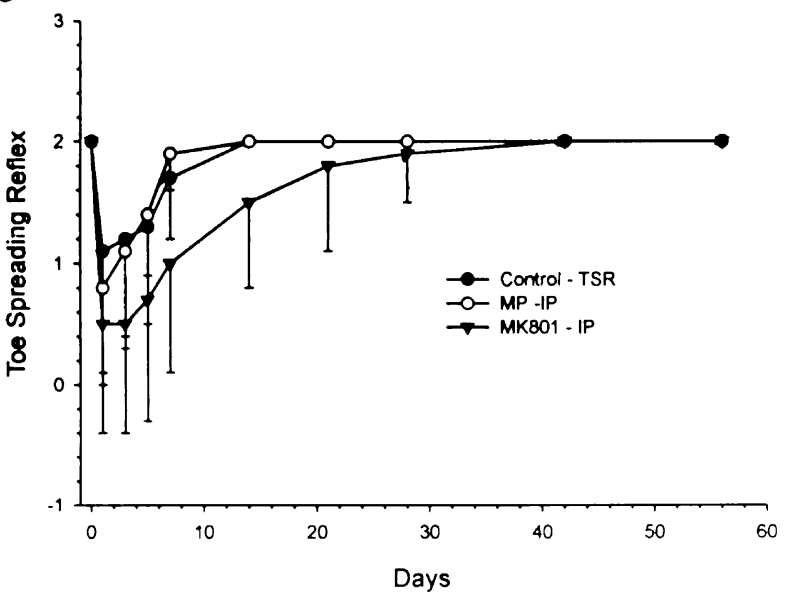

B
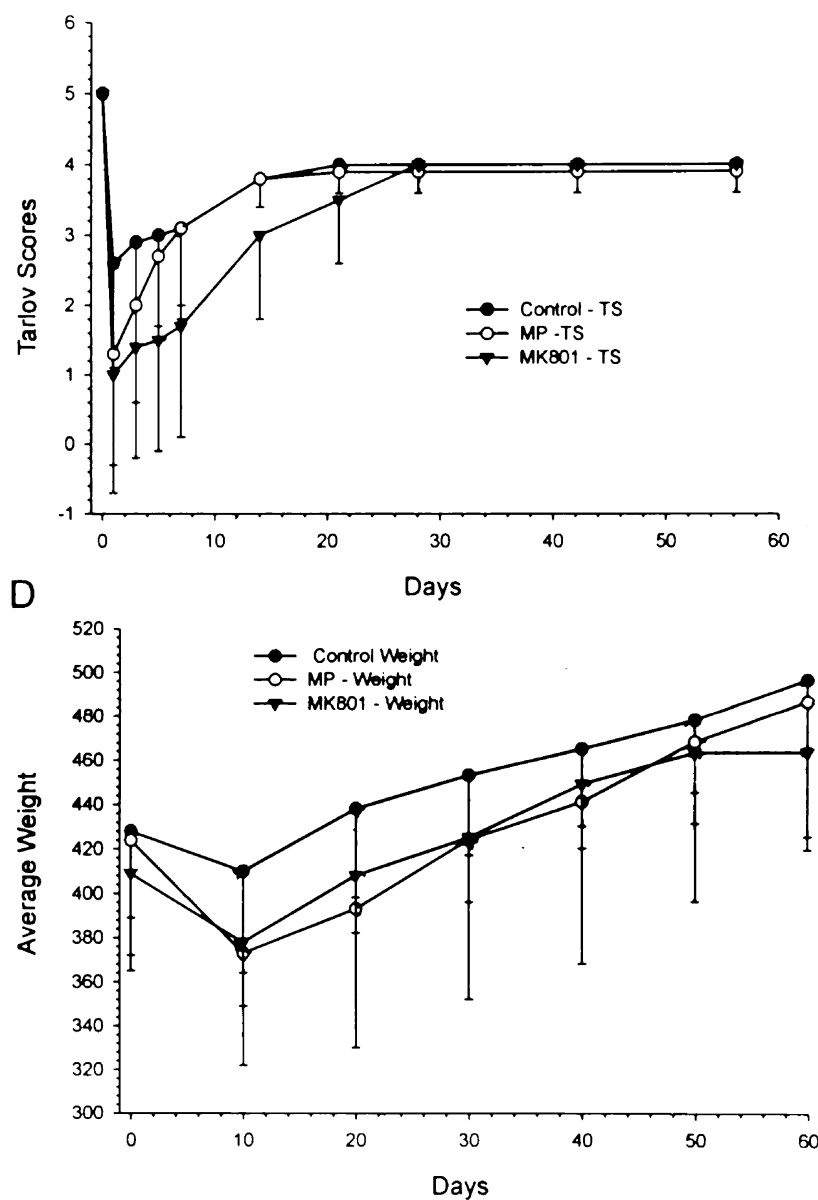

Figure 1 Effect of methylprednisolone $(30 \mathrm{mg} / \mathrm{kg})$ and MK-801 (30 mg/kg) on recovery of inclined plane scores (A), Tarlov scores $(\mathbf{B})$, Toe spreading reflex $(\mathbf{C})$ and Average weight (D) for 8 weeks after compression injury of thoracic spinal cord 
weight, IP and TS data. SPSS 9.0 was used for major analysis. Whenever Sphericity test for the homogeneity of covariance was significant, Greenhouse-Geisser adjustment was used for univariate repeated measure test. For TSR the Fisher's Exact test was used to compare the groups. Scores for the individual sections were averaged and statistically analyzed by the MannWhitney Rank Sum Test (Sigma Stat 1.0, SPSS Chicago, IL, USA). Differences at 0.05 level were considered significant.

\section{Results}

Figure 1 depicts the summary of the behavioral scores given to the control and the treated animals after the compressive injury. The recovery curves for the IP, TS, TSR, and weights assigned to the groups are shown in Figure $1 \mathrm{~A}-\mathrm{D}$. For the IP (Figure 1A), repeated measure analysis of variance was performed. There was no significant interaction between groups and IP measured on different days $F(18,216)=0.94(P>0.05)$. Greenhouse-Geiser correction factor was applied because of violation of the assumptions of homogeneity of covariance matrix for days 42 and 56 . The results were consistent showing no significant effect. Analysis of the TS (Figure 1B) showed no significant treatment effect at the 42nd and 56th day post-injury with repeated measure analysis of variance $(P>0.05)$. Analysis of cross-tabs tables of the TSR (Figure 1C) on days 42 and 56 also showed no significant difference between the TSR over the groups $(P>0.05)$. Finally, there was significant difference found between the weights of the three groups at baseline, 42, or 56 days post-injury $(P<0.05)$ (Figure 1D).

All specimens had some degree of cavitation and Wallerian degeneration located at one or more of the levels sampled in the cord. Cavitation involved both gray and white matter, but was generally most extensive in the former. Across the various levels, cavitation was generally most severe in the dorsal specimens, becoming less extensive at deeper levels. Figure 2 shows the mean histologic scores for $\mathrm{H} \& \mathrm{E}$ sections at each of the levels examined for the different groups. In group A and C, lesion scores were highest at the most superficial level, with declining scores in subsequent more ventral specimens. Specimens of Group B had larger malacic foci, as reflected by higher mean scores, and by the presence of larger lesions deeper in the spinal cord. Asterisks in Figure 2 indicate statistical differences between groups A and B for the second through fourth sectioning levels, but groups $\mathrm{B}$ and $\mathrm{C}$ were not significantly different.

When histological scores were averaged over the four levels, the mean of group B (mean score $2.80 \pm 0.21$ SEM) was significantly higher than that of group A $(1.68 \pm 325 ; P=0.0094)$. Although group C also had a lower mean $(1.97 \pm 0.51)$ than group $\mathrm{B}$, the two were not significantly different $(P=0.12)$ due to

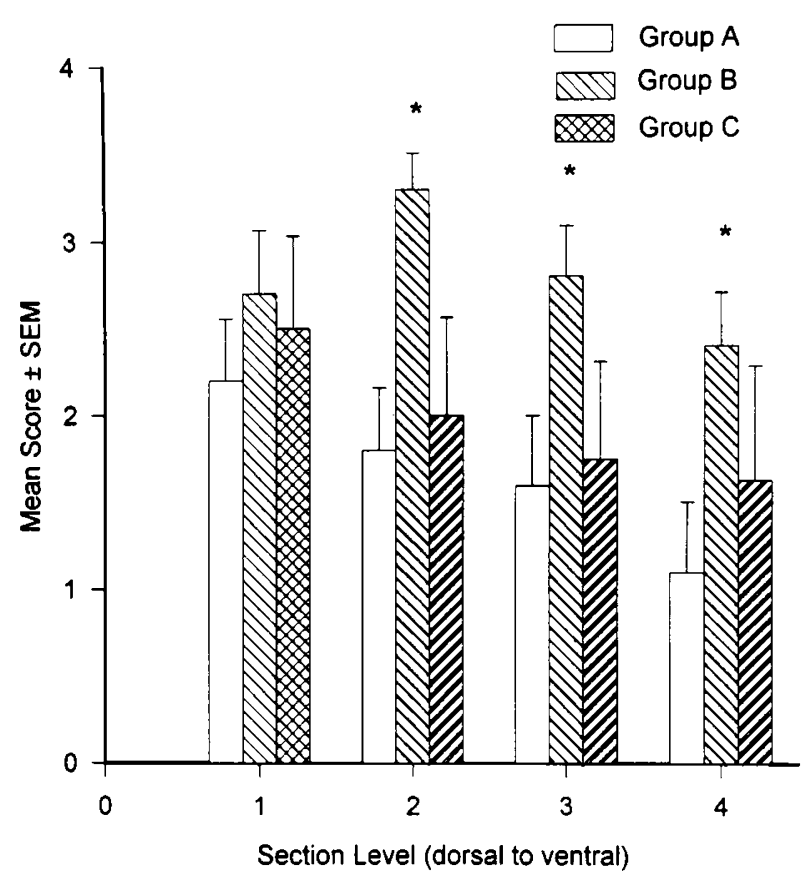

Figure 2 Mean histologic scores for each group ( \pm standard error of the mean) evaluated using longitudinal, HE-stained sections prepared at $100 \mu \mathrm{m}$ intervals. Section 1 is the most dorsal and section 4 the most ventral. Scores of $0-4$ possible at each level

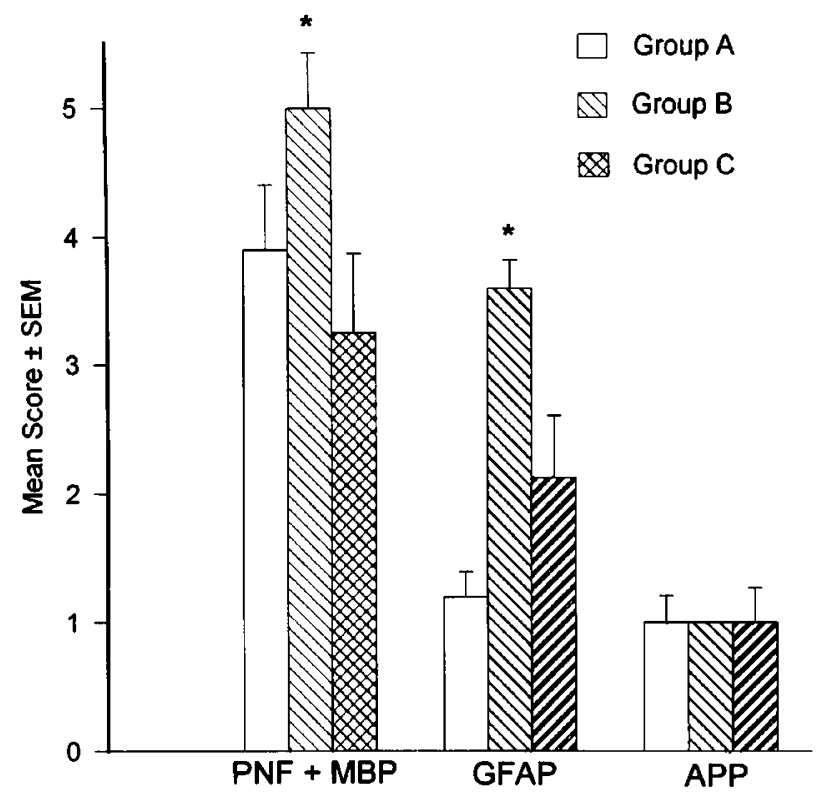

Figure 3 Mean histologic scores for various immunohistochemical stains taken from the most severely affected level of each spinal cord. Antigens include PNF (phosphorylated neurofilament), MBP (myelin basic protein), left; the scores from these two are combined because loss of both antigens is characteristic of WD. In the center bars, GFAP (glial fibrillary acidic protein) is specific for astrocytes. Right hand bars; APP (Beta-amyloid protein) 

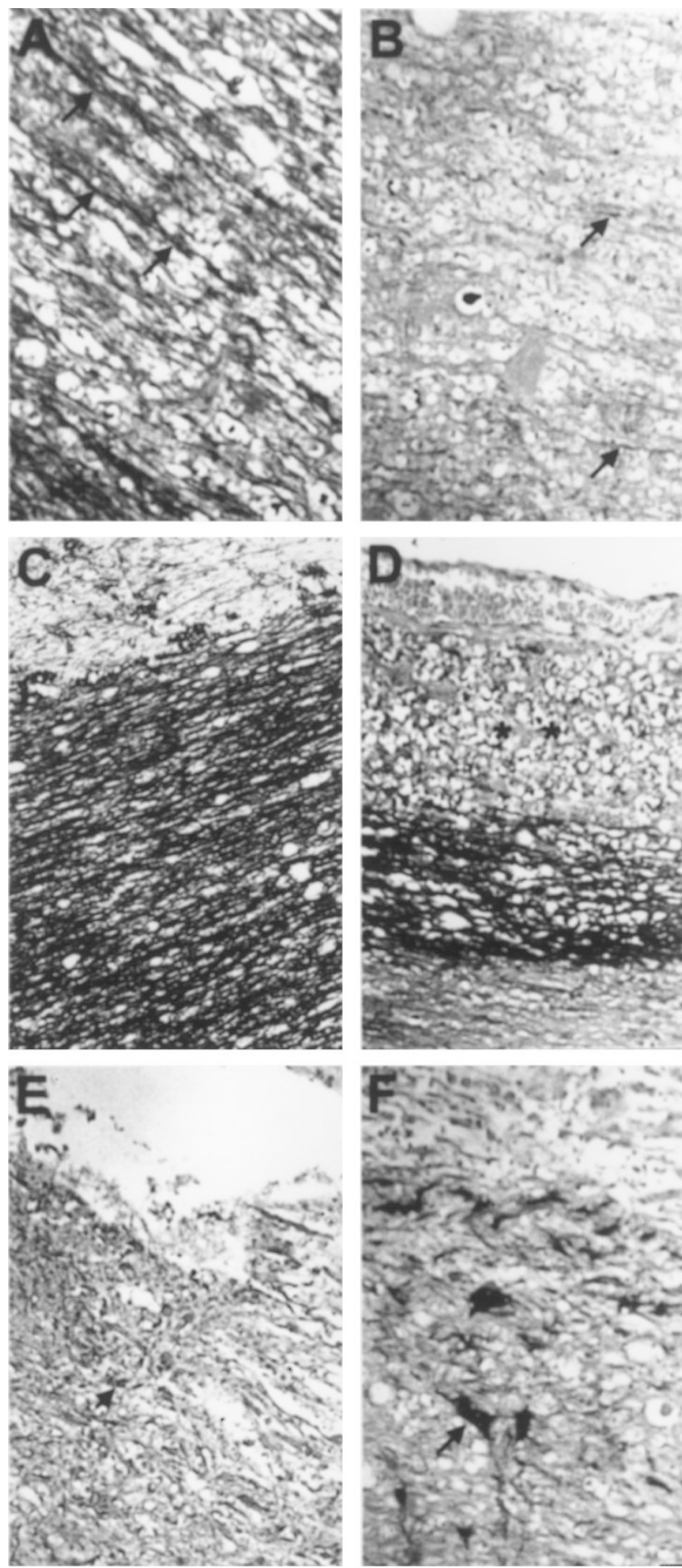

Figure 4 Immunohistochemically stained tissue demonstrating the distribution of specific antigens in mild $(\mathbf{A}, \mathbf{C}, \mathbf{E})$ and severe lesions $(\mathbf{B}, \mathbf{D}, \mathbf{F})$. PNF staining resulted in chromogen deposition on axons (arrows), which remained in relatively high numbers in low-score (2) specimens but are very few in number in one scored 4. MBP staining was similarly distributed. Asterisks in severely affected tissue (D) show a dramatic transition between severely and mildly affected tissue. GFAP staining of a mild lesion (score=2) shows variation in individual group $\mathrm{C}$ values. Groups $\mathrm{A}$ and $\mathrm{C}$ did not differ $(P=0.62)$.

Figure 3 summarizes immunohistochemical results, comparing mean scores of specimens from the various treatment groups stained with the different immunohistochemical markers. In all specimens, areas of WD were observed lateral, proximal and distal to cavitated foci in the cord. Because WD results in concomitant loss of stained PNF-axons in the tissue (Figure 4A,B) and by loss of MBP staining as well (Figure 4C,D). Therefore, for the purposes of data analysis, MBP and PNF loss scores were added and assessed together. Mean scores in treatment group B were highest. The mean of group B $(5.00 \pm 0.42)$ was greater than that of group $\mathrm{C}(3.25 \pm 0.62, P=0.03)$ but not that of group A $(3.90 \pm 0.50, P=0.11)$. Groups $\mathrm{A}$ and $\mathrm{C}$ were similar $(P=0.42)$.

Astrocytosis and astrocytic hyperplasia are common, nonspecific reactions to tissue injury in the nervous system, as reflected in increased astrocyte size and number. Some degree of astrocytosis was present around in most specimens. This change was least apparent in group A (Figure 4E), intermediate in group B and most extensive in group C. Astrocytes were of fibrillary morphology in group A. Gemistocytic astrocytes were found occasionally in six to eight specimens in group $\mathrm{C}$ and were numerous bordering
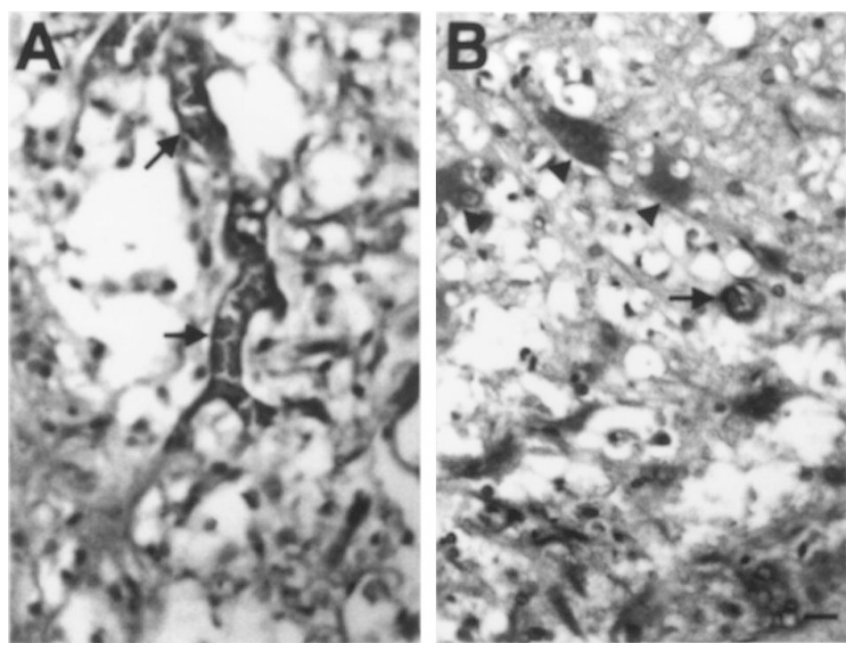

Figure $5(\mathbf{A}, \mathbf{B})$ Sections immunochemically stained for $\beta$ amyloid protein. The walls of small blood vessels (arrows, $\mathbf{A}, \mathbf{B})$ and the cytoplasm of gemistrocytes (arrowheads, B) are positive. Bar $=20 \mu \mathrm{m}$, both photographs are of similar magnification. Vector red chromogen with hematoxylin counterstain

increased density of predominantly fibrous astrocytes (arrow). In contrast, severely affected tissue (F) contains not only fibrous astrocytes (dorsal area) but also numerous larger (arrow). Bar, $\mathbf{E}=60 \mu \mathrm{m} ; \mathbf{A}-\mathbf{E}$ are of similar magnification). Bar, $\mathbf{F}=20 \mu \mathrm{m}$. Vector red chromogen with hematoxylin counterstain 
malacic areas in eight to 10 specimens in group C. Fibrillary astrocytosis was also more severe in these groups C (Figure 4F, arrow). Mean scores reflecting the extent and severity of astrocytosis were highest in group B $(3.60 \pm 0.22)$, compared to $1.20 \pm 0.20$ for group A $(P<0.001)$ and $2.13 \pm 0.48$ for group $\mathrm{C}$ $(P=0.029)$. Mean scores from group A did not differ from group $\mathrm{C}(P=0.11)$.

Beta-amyloid protein is expressed in traumatic injuries of various kinds. Only modest staining was observed in any of the specimens from this study, with no differences between groups. Positive staining was noted in the walls of blood vessels that crossed areas of cavitation (Figure 5A,B, arrows), and in the cytoplasm of gemistocytic astrocytes (arrowheads).

\section{Discussion}

In the present study, we have shown that neither blockade of NMDA receptors nor methylprednisolone improve recovery after compressive injury to adult rat spinal cord. With immunohistochemistry we characterized the presence of Wallerian degeneration, astrocytosis and presence of $\beta$-amyloid protein in thoracic spinal cord. However, the extent of behavioral neurological recovery was similar in the treated and control animals over the course of this study.

Glutamate receptor activation can increase the intracellular $\mathrm{Ca}^{2+}$ concentration in neurons and has been implicated in $\mathrm{Ca}^{2+}$-mediated cell death in a variety of neurodegenerative disorders. ${ }^{20,21}$ NMDA receptors are known to be highly calcium permeable. In the present study there was not a sustained improvement in behavioral neurological function with MK-801. Furthermore, these findings are in agreement with the previous studies, ${ }^{22,23}$ which found that NMDA antagonists did not ameliorate the extent of tissue loss after traumatic SCI.

Methylprednisolone (MP) given shortly after SCI significantly enhances recovery following injury. ${ }^{12,24}$ The effect was probably achieved by the reduction of lipid peroxidation or enhancement of spinal cord blood flow. Hall and his colleagues have reported a protective effect of MP in spinal cord ischemia resulting from spinal injury. ${ }^{24}$ In contrast, high doses of MP failed to improve recovery of motor or sensory function at 6 weeks and 6 months if administration of MP was given $8 \mathrm{~h}$ or more after injury. ${ }^{12}$ Recent review, which evaluated the literature from 1966 to 1999 suggested that high dose of MP should be excluded from consideration as an intervention for acute spinal cord injury ${ }^{15}$ because it does not improve neurological recovery after trauma. In an experimental study the effect of MP on vascular injury associated with SCI bolus injection of MP did not significantly affect the vascular injury. ${ }^{25}$ In the present study intraperitoneal injection of methylprednisolone $(30 \mathrm{mg} / \mathrm{kg})$ immediately after the injury and at 2,3 , 4, 5, 7 and 14 days post-injury also did not have any significant long-term beneficial effect as compared to control rats. The differences between previous studies and the present study may be the difference in injury weight drop versus compression injury. In the present study the injury was mild, and perhaps there was not enough difference to monitor changes. Further studies with severe injury could be more beneficial.

Immunohistochemistry was used in determining the extent of the damage around malacic spinal cord in this rat model of spinal trauma. Wallerian degeneration was the principal histologic lesion towards the pial margin to central malacic foci, and determining the extent of this change was important in determining the amount of morphologically disturbed tissue present. PNF antigen is present in normal axons throughout the nervous system. ${ }^{26}$ During Wallerian degeneration (WD), axonal fragmentation results in the degradation and disappearance of PNF staining. ${ }^{27}$ Fragmented axons are removed from various sites in the nervous system at different rates following WD, depending largely on axon caliber. ${ }^{28}$ However, there was no discernable difference between the degree of PNF staining loss in areas of WD between dorsal and ventral white tracts in our animals. The severe, longduration lesions in the rats resulted in excellent contrast between degenerate and normal white matter that allowed straightforward assessment of the extent of lesions.

Localization of MBP was also useful in detecting the myelin loss that occurs concomitantly with axonal fragmentation in WD. MBP is one of two markers commonly used to assess oligodendrocyte number and differentiation. A myelin-specific protein, MBP is predominantly in oligodendrocyte processes. An absence of staining implies demyelination but not necessarily oligodendrocyte loss. Expression of MBP is persistently absent in areas of traumatized spinal cord destined to undergo necrosis, while immunohistochemical staining remains but is confined to the cytoplasm of macophages. MBP signals are increased transiently between 2 and 14 days in viable tissue towards the pial margin of the spinal cord. ${ }^{29,30}$ MBP staining was minimal in the central malacic areas of our samples, perhaps because the animals were harvested late in the recovery period.

Both immature and mature oligodendrocytes express the immunohistochemically detectable enzyme 2'$3^{\prime}$-cyclic $3^{\prime}$ nucleotide phosphodiesterase (CNPase) in their cell bodies. Transiently increased numbers of CNPase-positive, MBP-negative oligodendrocytes have been observed towards the pial margin to areas of malacia following trauma ${ }^{29,30}$ with subsequent loss of staining in areas undergoing WD after a week. We had hoped to utilize this reagent to detect loss and/or attempted regeneration of oligodendrocytes towards the pial margin of the cord in our samples, but we were unsuccessful in staining our acid-decalcified tissues.

The most striking difference between animals with mild spinal cord lesions was the extent of astrocyte hypertrophy and proliferation. GFAP is a sensitive 
indicator of astrocyte perturbation in the hours to weeks following injury. ${ }^{31,32}$ Upregulation of this intermediate filament can be detected in as little as $1 \mathrm{~h}$ after injury, ${ }^{32}$ with persistence for weeks posttrauma. ${ }^{30,33}$ Large reactive (gemistrocytic) astrocytes were found in six to eight samples in group $\mathrm{C}$ and in eight to 10 specimens in group $\mathrm{B}$, indicating strong stimulation of astrocytes in these treatment groups.

Beta-amyloid protein is nonspecifically elaborated in response to various physical and hypoxic injuries to the nervous system and is utilized as a stable, reproducible marker for per acute trauma. ${ }^{34,35}$ Following experimental brain trauma, this protein can persist in small amounts for up to 1 year following experimental trauma. ${ }^{36} \mathrm{We}$ were surprised not to see more extensive staining in our sections, although a lack of sustained damage may have terminated elaboration or retention of this protein after the initial insult.

The presence of $\beta$-amyloid has recently been demonstrated to induce reactive astrocytes in vitro, although spinal cord astrocytes are thought to be less responsive to this protein than those from the hippocampus or cerebrum. ${ }^{37}$ Uptake and apparent phagocytosis of $\beta$-amyloid by astrocytes has been observed in chronic neurological conditions such as Alzheimer's disease, ${ }^{38}$ resulting in immunohistochemical staining, similar to that seen in gemistrocytes of some of our rats (Figure 5). Contact with $\beta$-amyloid can enhance GFAP expression in hippocampal astrocytes in vitro. ${ }^{39}$ Furthermore, some forms of $\beta$ amyloid can stimulate astrocytes to produce amyloidrelated peptides themselves. ${ }^{40}$ While, it is difficult to extrapolate to the in vitro situation, transient early expression of $\beta$-amyloid may have played a role in the increased GFAP expression observed in gemistocytes, even though end-stage amyloid staining is not striking in any of the rats.

\section{References}

1 Holtz A, Nystrom B, Gerdin B, Olsson Y. Neuropathological changes and neurological function after spinal cord compression in the rat. J Neurotrauma 1990; 7: 155-167.

2 Tator $\mathrm{CH}$, Fehlings MG. Review of the secondary injury theory of acute spinal cord trauma with emphasis on vascular mechanisms. J Neurosurg 1991; 75: 15-26.

3 Fehlings $\mathrm{MG}$, Tator $\mathrm{CH}$, Linden $\mathrm{RD}$. The relationships among the severity of spinal cord injury, motor and somatosensory evoked potentials and spinal cord blood flow. Electroencephalog Clin Neurophysiol 1989; 74: 241 - 259.

4 Faden AI, Simon RP. A potential role for excitotoxins in the pathophysiology of spinal cord injury. Ann Neurol 1988; 23: $623-626$.

5 Faden AI, Lemke MM, Simon RP, Noble LJ. N-methyl-Daspartate antagonists MK801 improves outcome following traumatic spinal cord injury in rats: behavioral, anatomic, and neurochemical studies. J Neurotrauma 1988; 5: $27-37$.

6 Faden AI, Ellison JA, Noble LJ. Effects of competitive and noncompetitive NMDA receptor antagonists in spinal cord injury. European J Pharmacol 1990; 175: $165-174$.
7 Panter SS, Yum SW, Faden AI. Alteration in extracellular amino acids after traumatic spinal cord injury. Ann Neurol 1990; 27: $96-99$.

8 Choi DW. Glutamate neurotoxicity in cortical cell culture is calcium dependent. Neurosci Lett 1985; 58: $293-297$.

9 Choi DW. Ionic dependence of glutamate neurotoxicity. $J$ Neurosci 1987; 7: 369-379.

10 Leach M. et al. BW619C89, a glutamate release inhibitor, protects against focal cerebral ischemic damage. Stroke 1993; 24: $1063-1067$.

11 Faden AI. Experimental neurobiology of central nervous system trauma. Crit Rev Neurobiol 1993; 7: 175-186.

12 Bracken MB et al. Methylprednisolone or naloxone treatment after acute spinal cord injury: 1-year follow-up data: results of the Second National Acute Spinal Cord Injury Study. $J$ Neurosurg 1992; 76: 23 -31.

13 Bracken MB et al. Administration of methylprednisolone for 24 or 48 hours or tirilazad mesylate for 48 hours in the treatment of acute spinal cord injury. Results of the Third National Acute Spinal Cord Injury Randomized Controlled Trial. National Acute Spinal Cord Injury Study. JAMA 1997; 277: 1597-1604.

14 Nesathurai S. Steroids and spinal cord injury: revisiting the NASCIS 2 and NASCIS 3 trials. J Trauma 1998; 45: 1088 - 1093.

15 Short DJ, El Masry WS, Jones PW. High-dose Methylprednisolone in the management of acute spinal cord - a systemic review from a clinical perspective. Spinal Cord 2000; 38: 273-286.

16 Rivlin AS, Tator $\mathrm{CH}$. Objective clinical assessment of motor function after experimental spinal cord injury in the rat. $J$ Neurosurg 1979; 47: $577-581$.

17 Gale K, Kerasidis H, Wrathall JD. Spinal cord contusion in the rat: behavioral analysis of functional neurologic impairment. Exp Neurol 1985; 88: $123-134$.

18 Tarlov IM. Spinal cord compression studies III. Time limits for recovery after gradual compression in dogs. Arch Neurol Psychiat 1954; 7: $588-597$

19 Haghighi SS et al. Effect of 4-aminopyridine and single dose methylprednisolone on functional recovery after a chronic spinal cord injury. Spinal Cord 1998; 36: 6-12.

20 Rothman SM, Olney JW. Excitotoxicity and the NMDA receptor. Trends Neurosci 1987; 10: 299-302.

21 Regan RF, Choi DW. Glutamate neurotoxicity in spinal cord cell culture. Neuroscience 1991; 43: 585-591.

22 Gomez-Pinilla F, Tram H, Cotman CW, Nieto-Sampedro M. Neuroprotective effect of MK801 and U-50488H after contusive spinal cord injury. Exp Neurol 1989; 104: 118 - 124.

23 Holtz A, Gerdin B. MK 801, an OBS N-methy-D-Aspartate channel blocker, does not improve the functional recovery nor spinal cord blood flow after spinal cord compression in rats. Acta Neurol Scand 1991; 84: $334-338$.

24 Hall ED, Wolf DL, Braughler JM. Effect of a single large dose of methylprednisolone sodium succinate on experimental posttraumatic spinal cord ischemia. Dose-response and time-action analysis. J Neurosurg 1984; 61: 124-130.

$25 \mathrm{Xu} \mathrm{J} \mathrm{ZXQ,} \mathrm{Hoggan} \mathrm{EL,} \mathrm{Perot} \mathrm{PL.} \mathrm{Protective} \mathrm{effect} \mathrm{of}$ methylprednisolone on vascular injury in rat spinal cord injury. J Neurotrauma 1992; 9: 245-253.

26 Rosenfeld J et al. Distribution of neurofilament antigens after axonal injury. J Neuropathol and Expt Neurol 1997; 46: 269-282.

27 Sherriff FE et al. Markers of axonal injury in postmortem human brain. Acta Neuropathol 1994; 88: 433-439.

28 Duchen LW. General pathology of neurons and neuroglia in Greenfield's Neuropathology, 5th: ed., JH Adams and LW Duchem, eds, Oxford University Press, New York, 1992.pp 168.

29 Bartholdi D, Schwab ME. Oligodendroglial reaction following spinal injury in rat: transient upregulation of MBP mRNA. Glia 1998; 23: $278-284$.

30 Morin-Richaud C, Feldblum S, Privat A. Astrocytes and oligodendrocytes reactions after total section of the rat spinal cord. Brain Res 1998; 783: 85-101. 
31 Farooque M, Badonic T, Olsson Y, Holtz A. Astrocytic reaction after graded spinal compression in rats: immunochemical studies on glial fibrillary acidic proteins and vimentin. J Neurotra 1995 ; 12: $41-52$.

32 Hadley SD, Goshgarian HG. Altered immunoreactivity for glial fibrillary acidic protein in astrocytes within $1 \mathrm{~h}$ after cervical spinal cord injury. Expt Neurol 1997; 146: $380-387$.

33 Baldwin SA, Broderick R, Blades DA, Scheff SW. Alterations in temporal/spatial distribution of GFAP- and vimentin-positive astrocytes after spinal cord contusion with the New York University spinal cord injury device. J Neurotrauma 1998; 15: $1015-1026$.

34 Shannon $\mathrm{P}$ et al. Axonal injury and the neuropathology of shaken baby syndrome. Acta neuropathol 1998; 95: 625-631.

35 Oehmichen $\mathrm{M}$ et al. Axonal injury - a diagnostic tool in forensic neuropathology? A review. Foren Sci International 1998; 95: $67-$ 83.

36 Pierce JES, Smith DH, Tronjanowski JQ, Mcintosh TK. Enduring cognitive, neurobehavioral and histopathological changes persist up to one year following severe experimental brain injury in rats. Neuroscience 1998; 87: 359-369.
37 Hoke A, Canning DR, Malemud CJ, Silver J. Regional differences in reactive gliosis induced by substrate bound betaamyloid. Expt Neurol 1994; 130: 56-66.

38 Funato $\mathrm{H}$ et al. Astrocytes containing amyloid beta-protein (A $\beta)$-positive granules are associated with A $\beta$-40-positive diffuse plaques in the aged human brain. Am J Pathol 1998; 152: $983-$ 992.

39 Meske V, Hamker U, Albert F, Ohm TG. The effects of $\beta /$ A4amyloid and its fragments on calcium homeostasis, glial fibrillary acidic protein and $\mathrm{S} 100 \beta$ staining, morphology and survival of cultured hippocampal astrocytes. Neuroscience 1998; 85: $1151-$ 1160

40 Moreno-Flores MT, Salinero O, Wandosell F. BA amyloid peptide $(25-35)$ induced APP expression in cultured astrocytes. $J$ Neurosci Res 1998; 52: $661-671$. 